OPEN

SUBJECT AREAS:

OPTICAL TWEEZERS

BIOPHOTONICS

Received

12 March 2014

Accepted

30 May 2014

Published

18 June 2014

Correspondence and requests for materials should be addressed to H.H. (haohe@tju.edu.

$\mathrm{cn})$

* Current address: Department of MicrobiologyImmunology, Feiberg School of Medicine, Northwestern University.

\section{All-optical regulation of gene expression in targeted cells}

\author{
Yisen Wang ${ }^{1}$, Hao He ${ }^{1,2}$, Shiyang Li*, Dayong Liv ${ }^{4}$, Bei Lan $^{3}$, Minglie Hu' , Youjia Cao ${ }^{3} \&$ Chingyue Wang'
}

'Ultrafast Laser Laboratory, Key Laboratory of Optoelectronic Information Technology (Ministry of Education), College of Precision Instrument and Optoelectronics Engineering, Tianjin University, Tianjin, 300072, P.R. China, ${ }^{2}$ Med-X Research Institute, School of Biomedical Engineering, Shanghai Jiaotong University, Shanghai, 200030, P.R. China, ${ }^{3}$ Key Laboratory of microbial functional genomics of Ministry of Education, College of Life Sciences, Nankai University, Tianjin, 300072, P.R. China, ${ }^{4}$ Department of Endodontics, School of Stomatology, Tianjin Medical University, Tianjin, 300072, P.R. China

Controllable gene expression is always a challenge and of great significance to biomedical research and clinical applications. Recently, various approaches based on extra-engineered light-sensitive proteins have been developed to provide optogenetic actuators for gene expression. Complicated biomedical techniques including exogenous genes engineering, transfection, and material delivery are needed. Here we present an all-optical method to regulate gene expression in targeted cells. Intrinsic or exogenous genes can be activated by a $\mathrm{Ca}^{2+}$-sensitive transcription factor nuclear factor of activated T cells (NFAT) driven by a short flash of femtosecond-laser irradiation. When applied to mesenchymal stem cells, expression of a differentiation regulator Osterix can be activated by this method to potentially induce differentiation of them. A laser-induced " $\mathrm{Ca}^{2+}$-comb" ( $\mathrm{LiCCo}$ ) by multi-time laser exposure is further developed to enhance gene expression efficiency. This noninvasive method hence provides an encouraging advance of gene expression regulation, with promising potential of applying in cell biology and stem-cell science.

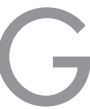
ene expression is essential for all living lives. Regulation of gene expression has been attracting great interests for better understanding of cellular processes, cell development, and cell physiology, as well as potential application in gene therapy, economical metabolic engineering and biopharmaceutical production. It has been previously found that specific patterns of cellular calcium oscillation can initiate signaling of gene expression ${ }^{1-4}$. Recently, progresses on optogenetics ${ }^{5-12}$, heat-activated promoters ${ }^{13}$, and gene/protein modification combined with nanoparticles and radio wave ${ }^{14}$ have successfully demonstrated controllable expression of some genes. However, all those methods include at least three complicated phases: 1) gene or protein engineering/ modification, 2) introduction of those bioengineered exogenous materials into cells, and 3) activation of those materials to express corresponding genes by some chemical or physical stimulation. Cells here play a role of "manufacturer" and the "import" of materials makes such methods complicated and invasive to cells. In this study, we developed a simple all-optical approach to regulate gene expression in cells by femtosecond laser noninvasively. Cells can produce proteins with their intrinsic genes activated by simple laser treatment without any "import".

Femtosecond lasers have been advancing biological researches with significant progresses ${ }^{15-17}$ by their unique advantages including high spatial resolution and good biological safety. It has been found that cellular $\mathrm{Ca}^{2+}$ level can be modulated by tightly focused femtosecond-laser irradiation ${ }^{18-20}$, where $\mathrm{Ca}^{2+}$ store in endoplasmic reticulum (ER) and $\mathrm{Ca}^{2+}$ channels in cytoplasm membrane are both involved ${ }^{21}$. Since intracellular $\mathrm{Ca}^{2+}$ plays the role of second messenger ${ }^{22}$, it is reasonable to design a scheme using femtosecond laser to regulate gene expression by taking advantage of a $\mathrm{Ca}^{2+}$-sensitive transcription factor. To this end, parameters of a Ti: Sapphire laser (repetition rate: $80 \mathrm{MHz}$, pulse duration: $75 \mathrm{fs}$ ) coupled in a confocal microscope (Supplementary Fig. S1a) were carefully adjusted to produce appropriate $\mathrm{Ca}^{2+}$ level in the targeted cell as the basic of all following works. As shown in Fig. 1a, a significant $\mathrm{Ca}^{2+}$ rise was excited by 0.3-s exposure of the focused femtosecond laser with a mean power of $40 \mathrm{~mW}$ a. It should be noted that high-power and long-duration irradiation of femtosecond laser can induce apoptosis (Supplementary Fig. S1b, c, and d).

\title{
Results
}

In this study, nuclear factor of activated T cells (NFAT) and its signaling pathway are selected for gene activation. We expected that with the stimulation of $\mathrm{Ca}^{2+}$ release induced by femtosecond laser, NFAT can be dephosphorylated by calcineurin. To verify this point, NFAT was genetically labeled with green fluorescent protein 

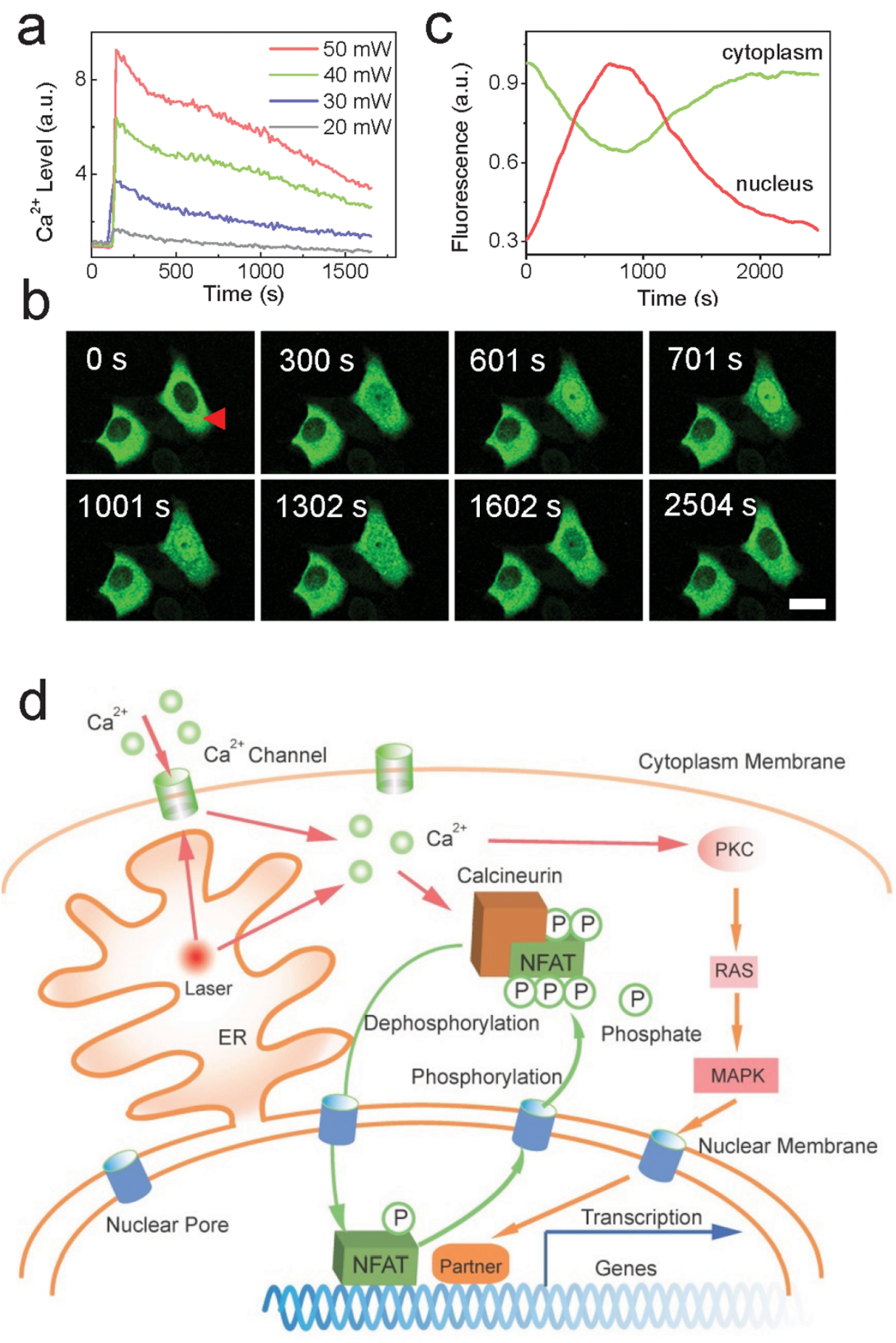

Figure 1 Scheme of all-optical regulation of gene expression method. (a). Typical cellular $\mathrm{Ca}^{2+}$ release after exposure of femtosecond laser at different powers $\left(\mathrm{n}=20\right.$ cells in each experiment). The power density is around $3 \times 10^{5} \mathrm{~W} / \mathrm{cm}^{2}$ at the mean power of $10 \mathrm{~mW}$. (b). GFP-tagged NFAT migration into nucleus after the stimulation of femtosecond laser. Bar: $10 \mu \mathrm{m}$. (c). Normalized fluorescence intensity of NFAT-GFP in cytosol and nucleus respectively. Significant migration of NFAT appeared at the 12th minute. (d). Proposed mechanism of femtosecond-laser induced gene transcription. The femtosecond laser exposure releases $\mathrm{Ca}^{2+}$ store in ER and provides stress to $\mathrm{Ca}^{2+}$ channel in cytoplasm membrane. Subsequently, NFAT is dephosphorylated and migrate into nucleus regulated by calcineurin. After the activation of corresponding genes, NFAT then moves out and is phosphorylated again. In the activation of gene expression, NFAT may cooperate with other transcriptional partners.

(GFP) to track its dynamic after laser treatment. It can be found that most NFAT distributed in cytoplasm and little in nucleus in normal times and translocated into nucleus after femtosecond-laser irradiation as we expected as shown in Fig. $1 \mathrm{~b}$ and $\mathrm{c}(\mathrm{n}=20$ cells in this experiment). In around 12 minutes, the translocation stopped and then NFAT began to exit from nucleus. Under different lasertreatment conditions, the speeds of NFAT translocation are different
(Supplementary Fig. S2). In following experiments, the optimal parameters of laser irradiation (0.3-s irradiation duration at $40-\mathrm{mW}$ power) were adopted, which could induce both efficient NFAT migration and good cell viability. As a control, if cellular $\mathrm{Ca}^{2+}$ was totally chelated at first, NFAT would not respond to laser treatment. The translocation of NFAT remarked the activation of NFAT transcription pathway to express corresponding genes ${ }^{23}$. Hence we pro- 


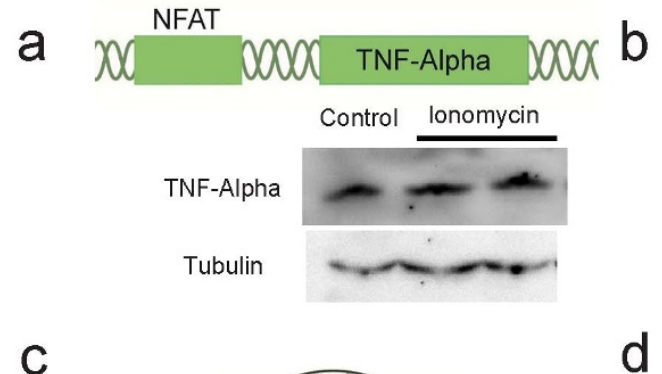

C

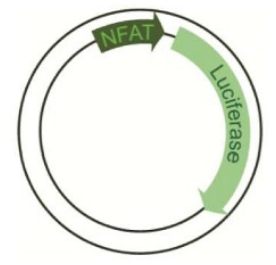

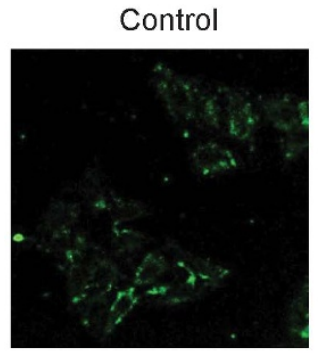

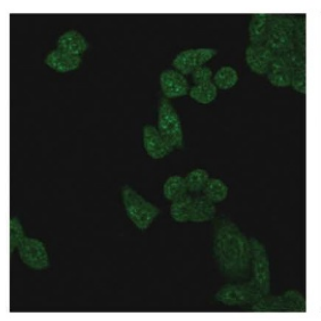

\section{Laser treated}
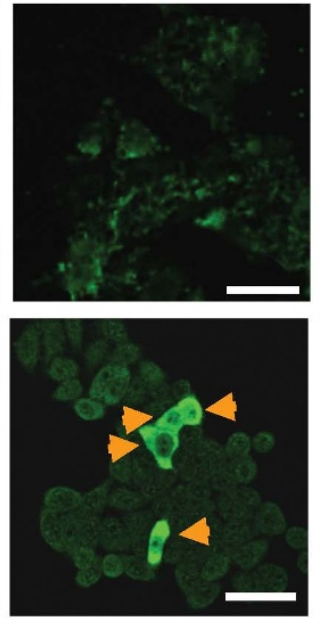
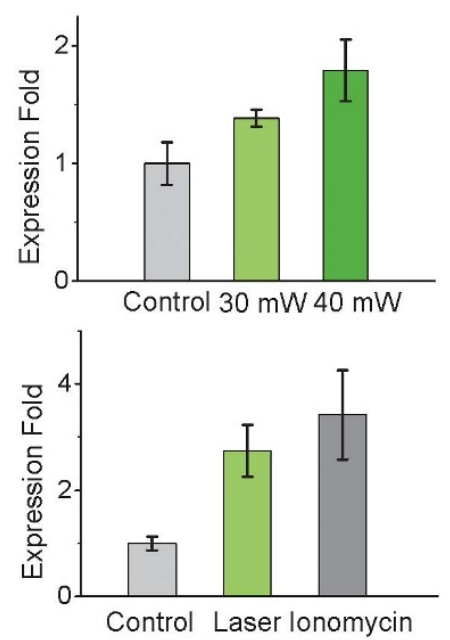

Figure $2 \mid$ Laser-regulated gene expression in HeLa cells. (a). TNF- $\alpha$ can be detected with or without ionomycin and $\mathrm{Ca}^{2+}$ treatment in HeLa cells as an endogenous gene tested by Western Blot. Tubulin: internal control in Western Blot. (b). Immunofluorescence microscopy of TNF- $\alpha$ in HeLa cells. Right: Upregulation of TNF- $\alpha$ in the cells with laser treatment $(\mathrm{n}=40$ cells in each experiment). The expression is more efficient if the laser-exposure duration is longer. Error bar: standard error of the mean. (c). Demonstration of NFAT-linked luciferase reporter plasmid that was transfected into HeLa cells. (d). Immunofluorescence microscopy of luciferase in HeLa cells. Around 14\% cells were transfected with NFAT-luciferase plasmid. If luciferase was expressed, there would be bright fluorescence in the cells indicated by yellow arrows. Right: The expression efficiency of luciferase by the optical method is close to the transfection efficiency determined by ionomycin and $\mathrm{Ca}^{2+}$-injection treatment. Error bar: standard error of the mean. Bar: $50 \mu \mathrm{m}$.

pose a reasonable scheme for optical regulation of gene expression as in Fig. 1d. The femtosecond laser irradiation at first induces significant rise of $\mathrm{Ca}^{2+}$ level, and then NFAT in cytoplasm is dephosphorylated. It can thus migrate into nucleus and activate expression of corresponding genes with or without some other transcriptional partners. After that, NFAT will be phosphorylated again and exits back into cytoplasm. In this way, such gene expression can be regulated optically without any biochemical treatments.

Here we measured the expression of tumor necrosis factor alpha (TNF- $\alpha$ ) in HeLa cells (Fig. 2a), an intrinsic and classic downstream gene of NFAT that plays an important role in immune response ${ }^{24}$, to verify our proposed scheme of laser-regulated gene expression. In experiments, HeLa cells were selected randomly and exposed for $0.1 \mathrm{~s}$ and $0.3 \mathrm{~s}$ respectively to the femtosecond laser. As shown in Fig. $2 b$, significant upregulation of TNF- $\alpha$ can be found in lasertreated cells ( $n=20$ cells in each experiment) after 6-hour incubation. The expression of TNF- $\alpha$ in cells exposed longer is more abundant because the NFAT activation can be more effective under intense laser stimulation.

As a proof of principle that this method can also activate exogenously designed genes, a plasmid with luciferase reporter gene under the promoter containing $3 \times$ NFAT binding site was then transfected to HeLa cells (Fig. 2c). The transfection efficiency was evaluated by global treatment of ionomycin and injection of $\mathrm{Ca}^{2+}$ for all cells. To test if such gene can be activated by our optical method, cells were also randomly selected and treated by femtosecond laser as described above ( $\mathrm{n}=170$ cells in this experiment). After 6-hour incubation, luciferase expression was found in most transfected cells as shown in Fig. 2 d. It can be found that the luciferase expression efficiency with laser treatment is very close to the transfection efficiency indicating most luciferase genes are expressed. This result reveals that exogenously designed genes can be effectively activated by this optical method.

Considering the universality of NFAT signaling, we show that this simple method can be applied to stem cells, which may potentially benefit regenerative medicine ${ }^{25}$ and treatment of autoimmune diseases $^{26}$. The laser-regulated TNF- $\alpha$ expression was then performed in mesenchymal stem cells (MSCs) from dental pulp tissue of human exfoliated deciduous teeth. Similarly, MSCs were randomly selected and directly irradiated by femtosecond laser for activation of TNF- $\alpha$ expression. The $\mathrm{Ca}^{2+}$ rise excited in MSCs is a little different with HeLa cells (Supplementary Fig. S3). After 3-day incubation, upregulation of TNF- $\alpha$ could be observed in laser-treated MSCs $(n=120$ cells in experiments) as shown in Fig. 3a.

Therefore, it is possible to induce stem-cell differentiation optically if this method can induce the expression of specific differentiation regulators. In this regard, we tried to use femtosecond laser to activate the expression of Osterix (Osx) in MSCs because it is a vital transcriptional factor that can induce MSCs to osteoblasts and bones $^{27}$. The expression of Osx can be activated by NFAT indirectly ${ }^{28,29}$ or combined with other ways, one of which is mitogenactivated protein kinase (MAPK) signaling pathway ${ }^{20,30,31}$ that can be also initiated by $\mathrm{Ca}^{2+}$ release ${ }^{32}$. Hence in theory the femtosecondlaser irradiation is possible to activate Osx expression. In our experiments, MSCs were then irradiated simply by the femtosecond laser to test this hypothesis. After 3-day incubation, significant expression of Osx in nucleus can be found in laser-treated MSCs as shown in Fig. 3b since the expressed Osx accumulated in nucleus which would then activate other specific genes as transcription factor to initiate the differentiation of MSCs to osteoblast. To further clarify this process, the accompanied expression of Runx2, the upstream gene of Osx that also regulated by NFAT and/or MAPK ${ }^{33}$, was then tested. As shown in Fig. $3 c$, Runx 2 could be detected in those laser-treated cells, indicating that the expression of Osx was activated by the proposed signaling pathway ${ }^{34}$.

This method was then furthered to improve the gene expression efficiency which can be affected by $\mathrm{Ca}^{2+}$ oscillation in cells ${ }^{3,4}$. We designed laser induced " $\mathrm{Ca}^{2+}$-comb" (Licco) in cells by treating them with multi-time femtosecond-laser irradiation to stimulate $\mathrm{Ca}^{2+}$ spikes. Each time after optical stimulation, $\mathrm{Ca}^{2+}$ was released from $\mathrm{Ca}^{2+}$ store at first and then slowly taken up by cells again until next stimulation. In this way, the "Licco" can mimic cellular $\mathrm{Ca}^{2+}$ oscillation (Supplementary Fig. S4) in cells. There are two critical factors to design Licco. First, the interval between two laser exposures should be longer than the duration of $\mathrm{Ca}^{2+}$ spike. To induce high-level but short-duration $\mathrm{Ca}^{2+}$ spikes, the exposure duration was then tuned to 

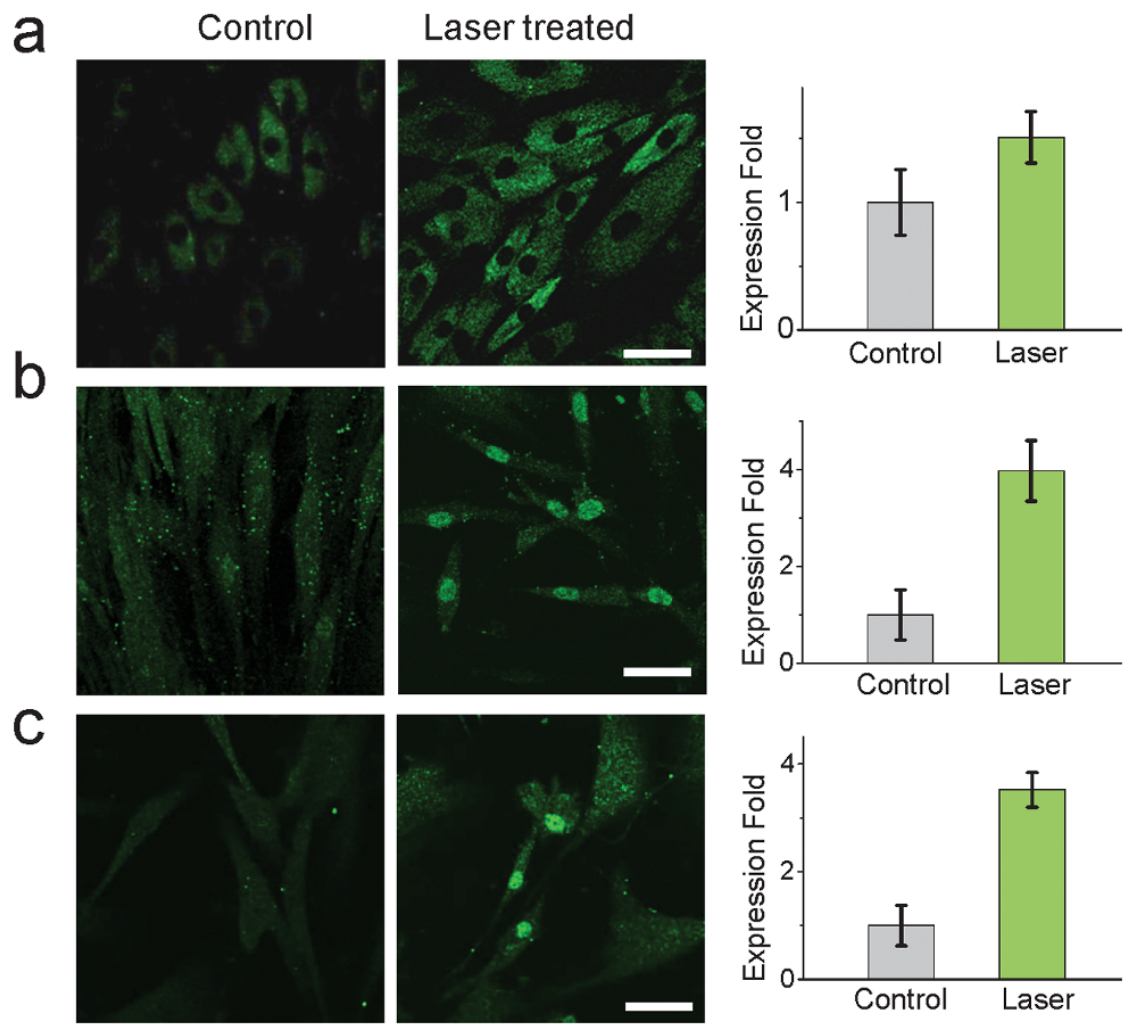

Figure 3 Laser-regulated gene expression in MSCs. (a). Immunofluorescence microscopy of TNF- $\alpha$ in MSCs. Right: Upregulation ratio of TNF- $\alpha$ in MSCs with laser treatment. (b). Immunofluorescence microscopy of Osx in MSCs. The intense fluorescence in nucleus indicates the expression of Osx since Osx accumulates in nucleus as a transcription factor. Right: Significant upregulation of Osx in laser-treated cells. (c). Immunofluorescence microscopy of Runx2 in MSCs. The intense fluorescence in nucleus indicates the expression of Runx2. Right: Significant upregulation of Runx2 in lasertreated cells. Error bar: standard error of the mean. Bar: $50 \mu \mathrm{m}$.

be $0.15 \mathrm{~s}$ with $35-\mathrm{mW}$ power. Second, the times of laser exposure should not exceed the safety condition, which was found in our experiments that most cells ( $>95 \%$ in 30 cells) would keep good membrane integrity after as more as 10-time laser exposure (each for $0.15 \mathrm{~s}$ ). Here a 6-spike Licco was performed in MSCs as shown in Fig. 4a. The fluorescence intensity decreased along each spike because the cell did not rest enough after each exposure to generate resembling $\mathrm{Ca}^{2+}$ release and the fluorescence emission efficiency of fluorophores as well as their re-combination with $\mathrm{Ca}^{2+}$ was also decreasing. This Licco method was then used to enhance gene expression in MSCs as shown in Fig. $4 \mathrm{~b}(\mathrm{n}=90$ cells in each experiment). The gene expression efficiency was improved, probably because the activation of the gene transcription mediated by laserinduced $\mathrm{Ca}^{2+}$ was enhanced.

\section{Discussion}

Different from optogenetics, this all-optical approach reported here provides a quite simple method to regulate gene expression noninvasively without any biochemical materials. Endo- and exogenous genes can be activated by short-time femtosecond-laser irradiation. The stress induced by this optical treatment to cells, which was usually thought to bring photodamage, is found to play a role of activating specific signaling pathways and finally expression of corresponding genes. Therefore, we do not need to introduce any specially designed materials or modification to cells for gene expression. In this regard, femtosecond laser is very promising to induce stem-cell differentiation by regulating corresponding differentiation regulators.

It is interesting that cells can express proteins under the simple stimulation. This means the molecular dynamics can be quite complicated when cells suffer stressors particularly physical damage like femtosecond-laser induced membrane perforation. Some important signaling proteins or transcription factors may be activated. Potentially some unknown stimulation or stress might induce cell reprogramming into pluripotent state by activating some unknown signaling pathways. Hence it is very attractive to further researches on molecular behaviors of cells under optical stimulation, which is more controllable and clean.

In conclusion, our results present an all-optical method for regulation of gene expression. The femtosecond laser here plays a role of initiation of gene transcription by activating the $\mathrm{Ca}^{2+}$-sensitive transcription factor NFAT. In this way, both endogenous and extraengineered genes can be activated by this simple method. It is encouraging that all-optically induced stem-cell differentiation can be thus potentially achieved since the expression of differentiation regulator Osx in MSCs can be regulated by this method. To be more efficient, the Licco method is developed to improve the efficiency of gene expression. Therefore, this noninvasive method is quite simple and noninvasive, which can be applied in the researches of gene expression, stem-cell differentiation, and immune responses.

\section{Methods}

Cell culture. HeLa cells were grown in Dulbecco's modified Eagle's medium supplemented with $10 \%$ FBS, $2 \mathrm{mM} \mathrm{L}$-glutamine, and $1 \%(\mathrm{v} / \mathrm{v})$ penicillin/ streptomycin at $37^{\circ} \mathrm{C}$ with $5 \% \mathrm{CO}_{2}$. Mesenchymal stem cells were isolated from dental pulp tissue of human exfoliated deciduous teeth after informed parental consent was obtained. The use of exfoliated human deciduous teeth was performed with the permission of the Ethical Committee of Tianjin Medical University. In brief, single cells were obtained by passing the cells through a $70 \mu \mathrm{m}$ strainer after digisted the pulp tissue in $3 \mathrm{mg} / \mathrm{ml}$ collagenase type I and $4 \mathrm{mg} / \mathrm{ml}$ dispase for $45 \mathrm{~min}$ at $37^{\circ} \mathrm{C}$. In this study, stem cells were cultured in alpha modified Eagle's medium supplemented with $10 \%$ FBS, 2 mM L-glutamine, and $1 \%(\mathrm{v} / \mathrm{v})$ penicillin/ streptomycin at $37^{\circ} \mathrm{C}$ with $5 \% \mathrm{CO}_{2}$ and at $3-5$ passages.

In experiments, cells were seeded in $35 \mathrm{~mm}$ petri dishes with a $0.17-\mathrm{mm}$ glass slide (Nest) for confocal microscopy. In all laser-treated experiments, petri dishes (ibidi) 


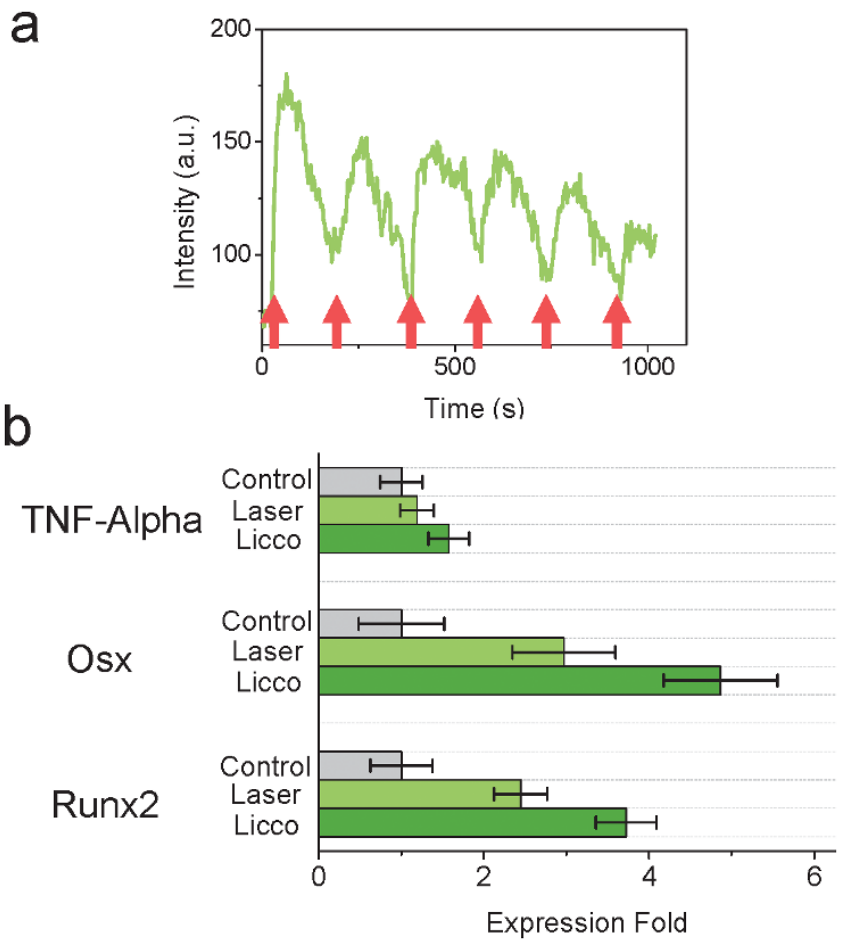

Figure $4 \mid$ Licco method can enhance the efficiency of gene expression. (a). $\mathrm{Ca}^{2+}$ spikes by Licco method. The frequency of Licco can be determined by the interval between two laser exposures and the duration of each $\mathrm{Ca}^{2+}$ spike. Red arrow: laser-exposure event. (b). Enhanced gene expression by Licco method with 6 spikes ( $\mathrm{n}=25$ cells in each experiment). The femtosecond laser was working at $35 \mathrm{~mW}$ and each time the exposure duration is $0.15 \mathrm{~s}$. Error bar: standard error of the mean.

with grid (numeric coordinates) on the bottom glass slides ( $0.17 \mathrm{~mm}$ thick) were used to localize the cells with laser irradiation.

Ethics statement. The experiments were carried out in accordance with the approved guidelines issued by the university and ethical committee. All biological materials and experiments were approved by the Ethical Committee of Nankai University, Tianjin University, and Tianjin Medical University.

Transfection. NFAT-GFP plasmid was supplied by Dr. Kazunori Kanemaru from University of Tokyo described as the reference ${ }^{35}$. The luciferase reporter plasmid, pNFAT-luc, was constructed with the $3 \mathrm{xNFAT}$ binding sequences derived from the IFN-gamma promoter ${ }^{36}$. NFAT-GFP and NFAT-Luciferase plasmids were transfected with a final concentration of $4 \sim 5 \mu \mathrm{g} / \mathrm{mL}$ by polyethylenimine (PEI) $(1 \mathrm{mg} / \mathrm{mL})$. Required amount of plasmid was dissolved in $100 \times$ Dulbecco's Modified Eagle Medium (DMEM), and then PEI was added in a volume to achieve PEI/DNA ratio at 5/1. The PEI-plasmid mixture was incubated at room temperature for 10 minutes and then added to cell culture medium drop by drop. After $24 \mathrm{~h}$, experiments were performed with those transfected cells.

Materials and confocal microscopy. Fluo-4/AM and JC-1 was purchased from Invitrogen (Life Technology) for $\mathrm{Ca}^{2+}$ labeling. The fluorophore staining procedures followed the protocol provided by the supplier. During confocal microscopy, green fluorescence was excited by a laser diode at $473 \mathrm{~nm}$ at $0.1 \mathrm{~mW}$. To minimize the photo-toxicity, confocal scanning was performed every $10 \mathrm{~s}$ to get one frame of image.

Optical setup and laser treatment. The Ti: Sapphire laser (pumped by Millenia V, Spectra Physics) was coupled into a confocal microscope (FV1000/IX81, Olympus) and focused by an objective $(60 \times$, water-immersed, N.A. $=1.2)$. The diameter of the laser focus is around $1 \mu \mathrm{m}$. The optical diagram and details are shown in Supplementary Fig. S1a. The output power of the femtosecond laser was attenuated to around $40 \mathrm{~mW}$ measured at specimen. The laser beam was at first blocked by a mechanical shutter. When a cell was selected, it would be moved to the position where the laser focus could stimulate the ER area of that cell by tuning the microscope stage. After that, the shutter would be open for laser treatment to the cell.

Immunofluorescence and Western blot. Immunofluorescence of TNF- $\alpha$, Luciferase, Osx and Runx2 was performed in HeLa and MS cells respectively with or without laser treatment. For TNF- $\alpha$, cells were treated with Brefeldin A Solution
(BioLegend, CA), fixed with $4 \%$ paraformaldehyde, and permeabilized with $0.5 \%$ Triton X-100. Immunofluorescence microscopy was then performed with FITC antihuman TNF- $\alpha$ mouse antibody (BioLegend, CA) by its standard protocol. To verify this method, another group of antibody (Supplementary Fig. S5), Anti-Human TNFa (RABBIT) Antibody and Anti-RABBIT IgG (H\&L) (GOAT) Antibody DyLight ${ }^{\mathrm{TM}}$ 488 Conjugated (Rockland) was also used. There would be no fluorescence if the first antibody was missing.

For luciferase, anti-firefly Luciferase rabbit antibody (Abcam, HK) and DyLightTM 488 conjugated anti-rabbit antibody (Rockland, PA) was used with the corresponding protocol. For RunX2, anti-RunX2 mouse antibody (Abcam, HK) and DyLight ${ }^{\circledR} 488$ goat polyclonal secondary antibody to mouse IgG-H\&L (Abcam, HK) was used following the protocol from the supplier. For Osx, anti-Sp7/Osterix rabbit antibody (Abcam, HK) and DyLight ${ }^{\circledR} 488$ goat anti-rabbit IgG secondary antibody (Abcam, HK) was used following the protocol from the supplier.

HeLa cells were washed, lysed, and incubated in ice. The protein was then extracted by centrifuging. The antibody Anti-Human TNF- $\alpha$ (Rabbit) antibody (Rockland, PA) was used for Western Blot.

Statistics. As TNF- $\alpha$ is an intrinsic gene of HeLa and MS cells, there existed a background expression in cells as in Fig. 2b. After laser stimulation, there would be an upregulation of TNF- $\alpha$ expression. Hence in this study, the epxression level of TNF- $\alpha$ was determined by the average fluorescence intensity of immunofluorescence of each cell at the same condition of immunofluorescence and confocal microscopy. This method was verified by comparing immunofluorescence with western blot of the control and ionomycin-treated cells.

The expression of luciferase in cells with NFAT-Luciferase plasmid transfected could be indicated by significant fluorescence by immunofluorescence of luciferase as in Fig. If (because this gene is not intrinsic gene of HeLa cells, there was no background expression). The cells with luciferase expressed could be then selected to caculate expression efficiency. In laser-treated cells, the efficiency was defined as the cells with luciferase expressed divided by all the laser-treated cells.

Similarly, cells with Osx or Runx2 expressed would exhibit significantly intense fluorescence in their nuclei as shown in Fig. 2 b and c. The expression efficiency was then defined as the cells with Osx/Runx 2 expressed in nuclues divided by the number of all cells with laser treatment.

1. Dolmetsch, R. E., Lewis, R. S., Goodnow, C. C. \& Healy, J. I. Differential activation of transcription factors induced by $\mathrm{Ca}^{2+}$ response amplitude and duration. Nature 386, 855-858 (1997).

2. Hardingham, G. E., Chawla, S., Johnson, C. M. \& Bading, H. Distinct functions of nuclear and cytoplasmic calcium in the control of gene expression. Nature 385, 260-265 (1997)

3. Dolmetsch, R. E., Xu, K. \& Lewis, R. S. Calcium oscillations increase the efficiency and specificity of gene expression. Nature 392, 933-936 (1998).

4. Li, W., Llopis, H., Whitney, M., Zlokarnik, G. \& Tsien, R. Y. Cell-permeant caged $\mathrm{Ins}_{3}$ ester shows that $\mathrm{Ca}^{2+}$ spike frequency can optimize gene expression. Nature 392, 936-941 (1998).

5. Deisseroth, K. Optogenetics. Nat. Meth. 8, 26-29 (2011).

6. Konermann, S. et al. Optical control of mammalian endogenous transcription and epigenetic states. Nature 500, 472-476 (2013).

7. Yazawa, M., Sadaghiani, A. M., Hsueh, B. \& Dolmetsch, R. E. Induction of proteinprotein interactions in live cellsusing light. Nat. Biotech. 27,941-945(2009).

8. Strickland, D. et al. TULIPs: tunable, light-controlled interacting protein tags for cell biology. Nat. Meth. 9, 379-384 (2012).

9. Kennedy, M. J. et al. Rapid blue-light-mediated induction of protein interactions in living cells. Nat. Meth. 7, 973-975 (2010).

10. Ye, H., Daoud-El Baba, M., Peng, R. W. \& Fussenegger, M. A synthetic optogenetic transcription device enhances blood-glucose homeostasis in mice. Science 332, 1565-1568 (2011).

11. Polstein, L. R. \& Gersbach, C. A. Light-inducible spatiotemporal control of gene activation by customizable zinc finger transcription factors. J. Am. Chem. Soc 134, 16480-16483 (2012).

12. Bugaj, L. J., Choksi, A. T., Mesuda, C. K., Kane, R. S. \& Schaffer, D. V. Optogenetic protein clustering and signaling activation in mammalian cells. Nat. Meth. 10, 249-252 (2013).

13. Kamei1, Y. et al. Infrared laser-mediated gene induction in targeted single cells in vivo. Nat. Meth. 6, 79-81 (2009).

14. Stanley, S. A. et al. Radio-Wave Heating of Iron Oxide Nanoparticles Can Regulate Plasma Glucose in Mice. Science 336, 604-608 (2012).

15. Tirlapur, U. K. \& König, K. Targeted transfection by femtosecond laser. Nature 418, 290-291 (2002).

16. Yanik, M. F. et al. Functional regeneration after laser axotomy. Nature 432, 822 (2004).

17. Nishimura, N. et al. Targeted insult to subsurface cortical blood vessels using ultrashort laser pulses: three models of stroke. Nat. Meth. 3, 99-108 (2006).

18. Zhao, Y. et al. Characteristics of calcium signaling in astrocytes induced by photostimulation with femtosecond laser. J. Biomed. Opt. 15, 035001 (2010).

19. He, H., Kong, S. K. \& Chan, K. T. Identification of source of calcium in HeLa cells by femtosecond laser excitation. J. Biomed. Opt. 15, 057010 (2010). 
20. Iwanaga, S. et al. Location-dependent photogeneration of calcium waves in HeLa cells. Cell Biochem. and Biophys. 45, 167-176 (2006).

21. He, H. et al. Manipulation of cellular light from green fluorescent protein by a femtosecond laser. Nat. Phot. 6, 651-656 (2012).

22. Berridge, M., Bootman, M. \& Lipp, P. Calcium - a life and death signal. Nature 395, 645-648 (1998).

23. Crabtree, G. R. \& Olson, E. N. NFAT Signaling: Choreographing Review the Social Lives of Cells. Cell 109, S67-S79 (2002).

24. Müller, M. R. \& Rao, A. NFAT, immunity and cancer: a transcription factor comes of age. Nat. Rev. Immu. 10, 645-656 (2010).

25. Frenette, P., Pinho, S., Lucas, D. \& Scheiermann, C. Mesenchymal Stem Cell: Keystone of the Hematopoietic Stem Cell Niche and a Stepping-Stone for Regenerative Medicine. Annu. Rev. Immunol. 31, 285-316 (2013).

26. Aggarwal, S. \& Pittenger, M. F. Human mesenchymal stem cells modulate allogeneic immune cell responses. Blood 105, 1815-1822 (2005).

27. Nakashima, K. et al. The Novel Zinc Finger-Containing Transcription Factor Osterix Is Required for Osteoblast differentiation and Bone Formation. Cell 108, 17-29 (2002).

28. Koga, T. et al. NFAT and Osterix cooperatively regulate bone formation. Nat. Med. 11, 880-885 (2005).

29. Alexopoulos, A. et al. Bone regulatory factors NFATc1 and Osterix in human calcific aortic valves. Int. J. Cardiol. 139, 142-149 (2010).

30. Celil, A. B. \& Campbell, P. G. BMP-2 and insulin-like growth factor-i mediate Osterix (Osx) expression in human mesenchymal stem cells via the MAPK and Protein Kinase D signaling pathways. J. Biol. Chem. 280, 31353-31359 (2005).

31. Kanno, T., Takahashi, T., Tsujisawa, T., Ariyoshi, W. \& Nishihara, T. J. Cell. Biochem. 101, 1266-1277 (2007).

32. Clapham, D. E. Calcium Signaling. Cell 131, 1047-1058 (2007).

33. Nishio, Y. et al. Runx2-mediated regulation of the zinc finger Osterix/Sp7 gene. Gene 372, 62-70 (2006).

34. Deschaseaux, F., Sensébé, L. \& Heymann, D. Mechanisms of bone repair and regeneration. Trends Mol. Med. 15, 417-429 (2009).

35. Tomida, T., Hirose, K., Takizawa, A., Shibasaki, F. \& Iino, M. NFAT functions as a working memory of $\mathrm{Ca}^{2+}$ signals in decoding $\mathrm{Ca}^{2+}$ oscillation. the EMBO journal 22, 3825-3832 (2003).
36. Underhill, D. M. et al. The Roles of Nuclear Factor of Activated T Cells and YingYang 1 in Activation-induced Expression of the Interferon- $\gamma$ Promoter in T Cells. J. Biol. Chem. 273, 34775-34783 (1998).

\section{Acknowledgments}

We thank Dr. Kazunori Kanemaru from University of Tokyo for the NFAT-GFP plasmid. Dr. Wenqin Wang from Harvard University and Dr. Keisuke Goda from University of Tokyo for effective discussions. This work was supported by grants from National Natura Science Foundation of China (NSFC) 61108080 to H.H., 81171556 to Y.C., 81371109 to D.L., and Foundation of Tianjin Educational Committee (Grant No. 20110139 to D.L.).

\section{Author contributions}

H.H. proposed the idea. H.H., Y.W. and S.L. together designed experiments. Y.W. performed experiments. D.L., S.L. and B.L. prepared and supported biological samples. H.H., Y.W., D.L. and Y.C. analyzed data. H.H. wrote the manuscript. Y.C., M.H. and C.W. supervised the research. All authors contributed to the discussion and feedback to the manuscript.

\section{Additional information}

Supplementary information accompanies this paper at http://www.nature.com/ scientificreports

Competing financial interests: The authors declare no competing financial interests.

How to cite this article: Wang, Y. et al. All-optical regulation of gene expression in targeted cells. Sci. Rep. 4, 5346; DOI:10.1038/srep05346 (2014).

This work is licensed under a Creative Commons Attribution-NonCommercialNoDerivs 4.0 International License. The images or other third party material in this article are included in the article's Creative Commons license, unless indicated otherwise in the credit line; if the material is not included under the Creative Commons license, users will need to obtain permission from the license holder in order to reproduce the material. To view a copy of this license, visit http:// creativecommons.org/licenses/by-nc-nd/4.0/ 